\title{
Numerical solutions for a Stefan problem
}

\author{
Hatice Karabenli, Alaattin Esen and E.Nesligul Aksan \\ Department of Mathematics, Inonu University, Malatya, Turkey \\ Received: 23 March 2016, Accepted: 4 May 2016 \\ Published online: 30 October 2016.
}

\begin{abstract}
The initial version of a Stefan problem is the melting of a semi-infinite sheet of ice. This problem is described by a parabolic partial differential equation along with two boundary conditions on the moving boundary which are used to determine the boundary itself and complete the solution of the differential equation. In this paper firstly, we use variable space grid method, boundary immobilisation method and isotherm migration method to get rid of the trouble of the Stefan problem. Then, collocation finite element method based on cubic B-spline bases functions is applied to model problem. The numerical schemes of finite element methods provide a good numerical approximation for the model problem. The numerical results show that the present results are in good agreement with the exact ones.
\end{abstract}

Keywords: Stefan problems, variable space grid method, boundary immobilisation method, isotherm migration method, collocation finite element method.

\section{Introduction}

Many problems in various areas of applied mathematics and engineering can be modelled as partial differential equations posed in domains whose boundaries are to be determined as part of the solution. Such problems are usually referred to as moving boundary problems. Base of Stefan problem was started with reference to early work of J. Stefan [1] who is interested in melting of the polar ice cap. We encounter these type of problems in wide-ranging applications physical and biological sciences, engineering, metallurgy, soil mechanics, decision and control theory. In these mentioned areas, the material undergoes to changing phase with a moving boundary that has to be determined as part of the solution. As a result, Stefan problems are non-linear problems and thus have the limited analytical solutions. Due to the shortage of the analytical solutions, numerical methods have been used more commonly [2].

There are two numerical techniques to obtain the solution of the Stefan problems. The first one is the front-tracking method, in which the position of the moving boundary is continuously tracked by some iterative procedure. Variable space grid method [3,4], variable time step method [5] and heat balance integral method [6] are examples of tracking methods for the moving boundary. The other approach is to use a front-fixing methods. Boundary immobilisation method [7], isotherm migration method [8,9,10] and enthalpy method [11] are alternatives to fix the moving boundary.

In this paper, we are going to concern with the melting of a semi-infinite sheet of ice, initially at the melting temperature taken to be zero. Firstly, we are going to apply variable space grid method, boundary immobilisation method and isotherm migration method. Then, we are going to obtain numerical solutions with finite element method (FEM) based on cubic B-splines. The computational results of temperature distribution, the position of the moving boundary and the velocity of moving boundary are given in the section 8 . 


\section{Model problem}

In the one-dimensional Stefan problem, the function $U(x, t)$ is temperature distribution governed by the heat equation

$$
\frac{\partial U}{\partial t}=\frac{\partial^{2} U}{\partial x^{2}}, \quad 0<x<s(t), \quad t>0
$$

subject to

$$
U(0, t)=1, \quad U(s(t), t)=0, \quad t>0
$$

boundary conditions where $U(0, t)$ is the constant surface temperature. Also, one further condition is needed on the moving interface to determine the position of the interface itself which is known as Stefan condition. In this problem Stefan condition is

$$
\frac{d s(t)}{s t}=-S t e \frac{\partial U}{\partial x}, \quad x=s(t), \quad t>0
$$

with the initial condition

$$
s(0)=0
$$

where $s(t)$ is the position of the moving boundary and Ste $=\frac{c \Delta t}{L}$ is the Stefan number, $c$ is the specific heat capacity of liquid, $\Delta t$ is a reference temperature and $L$ is the latent heat.

The model problem has the exact solution for temperature distribution and the moving boundary

$$
\begin{gathered}
U(x, t)=1-\frac{\operatorname{erf}(x / 2 \sqrt{t})}{\operatorname{erf}(\lambda)}, 0 \leq x \leq s(t), \quad t>0 \\
s(t)=2 \lambda \sqrt{t}, \quad t \geq 0
\end{gathered}
$$

where parameter $\lambda$ known as melting/solidification parameter is root of the transcendental equation

$$
\gamma \sqrt{\pi} \lambda e^{\lambda^{2}} \operatorname{erf}(\lambda)=1
$$

The exact solution is used to initialize the numerical computations and compare the numerical results for temperature distribution, the velocity of moving boundary and the position of moving boundary.

This problem has been recently solved by Savovic' \& Caldwell \& Kwan [12] with employing the nodal integral method (NIM) and finite difference method (FDM) constructed on boundary immobilisation method. We have compared the numerical solutions reached in reference [12] and the present solutions obtained using finite element method.

\section{Cubic B-splines collocation finite element method}

Let us divide interval $[a, b]$ into $N$ uniform elements consisting of the knots $x_{m}$ such as $a=x_{0}<x_{1}<\ldots<x_{N}=b$. Cubic B-splines $\phi_{m}$ spanning $[a, b]$ are $\phi_{-1}, \phi_{0}, \phi_{1}, \ldots, \phi_{N+1}$. So $U_{N}(x, t)$ approximate solution for $U(x, t)$ can be written as

$$
U_{N}(x, t)=\sum_{m=-1}^{N+1} \delta_{m}(t) \phi_{m}(x)
$$

where $\phi_{m}$ are trial functions given following expressions and $\delta_{m}$ are time-dependent variables which will be determined boundary and collocation conditions for the Stefan problem. The cubic B-spline bases functions are defined by the 
following relationship

$$
\phi_{m}(x)=\frac{1}{h^{3}} \begin{cases}\left(x-x_{m-2}\right)^{3}, & {\left[x_{m-2}, x_{m-1}\right]} \\ h^{3}+3 h^{2}\left(x-x_{m-1}\right)+3 h\left(x-x_{m-1}\right)^{2}-3\left(x-x_{m-1}\right)^{3}, & {\left[x_{m-1}, x_{m}\right]} \\ h^{3}+3 h^{2}\left(x_{m+1}-x\right)+3 h\left(x_{m+1}-x\right)^{2}-3\left(x_{m+1}-x\right)^{3}, & {\left[x_{m}, x_{m+1}\right]} \\ \left(x_{m+2}-x\right)^{3}, & {\left[x_{m+1}, x_{m+2}\right]} \\ 0, & \text { otherwise }\end{cases}
$$

where $\Delta x=h=x_{m}-x_{m-1}$ for all $m, m=-1,0,1, \ldots, N+1$.

The cubic spline $\phi_{m}$ and its principle derivatives $\phi_{m}^{\prime}$ and $\phi_{m}^{\prime \prime}$ disappear the outside of the interval $\left[x_{m-2}, x_{m+2}\right]$. We can tabulate values of the functions $\phi_{m}, \phi_{m}^{\prime}$ and $\phi_{m}^{\prime \prime}$ at the knots $x_{m}$ as the following table

Table 1: B-Spline values at the knots.

\begin{tabular}{cccccc}
\hline$x$ & $x_{m-2}$ & $x_{m-1}$ & $x_{m}$ & $x_{m+1}$ & $x_{m+2}$ \\
\hline$\phi_{m}$ & 0 & 1 & 4 & 1 & 0 \\
$\phi_{m}^{\prime}$ & 0 & $-\frac{3}{h}$ & 0 & $\frac{3}{h}$ & 0 \\
$\phi_{m}^{\prime \prime}$ & 0 & $\frac{6}{h^{2}}$ & $-\frac{12}{h^{2}}$ & $\frac{6}{h^{2}}$ & 0 \\
\hline
\end{tabular}

Now, we can define finite element approximation of nodal values for $U, U^{\prime}$ and $U^{\prime \prime}$ at the knot $x_{m}$

$$
\begin{aligned}
U & =U\left(x_{m}\right)=\delta_{m-1}+4 \delta_{m}+\delta_{m+1} \\
U^{\prime} & =U^{\prime}\left(x_{m}\right)=\frac{3}{h}\left(-\delta_{m-1}+\delta_{m+1}\right) \\
U^{\prime \prime} & =U^{\prime \prime}\left(x_{m}\right)=\frac{6}{h^{2}}\left(\delta_{m-1}-2 \delta_{m}+\delta_{m+1}\right),
\end{aligned}
$$

\section{Variable space grid method}

Murray and Landis [3] kept the number of space intervals between $x=0$ and $x=s(t)$ constant, equal to $N$, for all time. As a result, the moving boundary is always on the $N^{t h}$ grid line. In that case, the grid size must be $x=s(t) / N$ which is changed with the time.

For the line $x_{i}$, partial differentiation with respect to time $t$ is given by the following equation

$$
\left.\frac{\partial U}{\partial t}\right|_{i}=\left.\left.\frac{\partial U}{\partial x}\right|_{t} \frac{d x}{d t}\right|_{i}+\left.\frac{\partial U}{\partial t}\right|_{x}
$$

and at the $i^{t h}$ grid point variation of $\frac{d x}{d t}$ is given as

$$
\frac{d x_{i}}{d t}=\frac{x_{i}}{s(t)} \frac{d s}{d t}
$$

By substituting (12) into (11) the one dimensional heat equation becomes

$$
\left.\frac{\partial U}{\partial t}\right|_{i}=\left.\frac{x_{i}}{s(t)} \frac{d s}{d t} \frac{\partial U}{\partial x}\right|_{t}+\left.\frac{\partial U}{\partial t}\right|_{x}
$$


and dimensionless Stefan problem turns into the following equation

$$
\frac{\partial U}{\partial t}=\frac{x_{i}}{s(t)} \frac{d s}{d t} \frac{\partial U}{\partial x}+\frac{\partial^{2} U}{\partial x^{2}}, \quad 0<x<s(t)
$$

subject to (2)-(4) boundary and initial conditions.

By substituting $U$ and its derivatives $U^{\prime}, U^{\prime \prime}$ given with equation (10) into equation (13), we obtain the following equation

$$
\dot{\delta}_{m-1}+4 \dot{\delta}_{m}+\dot{\delta}_{m+1}=x_{m}^{n} \frac{(\dot{s})^{n}}{s^{n}} \frac{3}{h}\left(-\delta_{m-1}+\delta_{m+1}\right)+\frac{6 \alpha}{h^{2}}\left(\delta_{m-1}-2 \delta_{m}+\delta_{m+1}\right) .
$$

And using Crank-Nicolson approach and forward difference approximation for two time levels $n$ and $n+1$, such as

$$
\delta_{m}=\frac{\delta_{m}^{n+1}+\delta_{m}^{n}}{2}, \quad \dot{\delta}_{m}=\frac{\delta_{m}^{n+1}-\delta_{m}^{n}}{\triangle t}
$$

where $\delta_{m}^{n}$ are the parameters at the time $n \Delta t$ and "." demonstrates derivative respected to time. We have the finite element system by using some mathematical operations

$$
\alpha_{m 1} \delta_{m-1}^{n+1}+\alpha_{m 2} \delta_{m}^{n+1}+\alpha_{m 3} \delta_{m+1}^{n+1}=\alpha_{m 4} \delta_{m-1}^{n}+\alpha_{m 5} \delta_{m}^{n}+\alpha_{m 6} \delta_{m+1}^{n}, \quad m=0,1, \ldots, N
$$

where the coefficients are

$$
\begin{aligned}
\alpha_{m 1}=1+x_{m}^{n} \frac{3 \Delta t(\dot{s})^{n}}{2 \Delta x^{n} s^{n}}-\frac{3 \Delta t}{\left(\Delta x^{n}\right)^{2}}, & \alpha_{m 4}=1-x_{m}^{n} \frac{3 \Delta t(\dot{s})^{n}}{2 \Delta x^{n} s^{n}}+\frac{3 \Delta t}{\left(\Delta x^{n}\right)^{2}} \\
\alpha_{m 2}=4+\frac{6 \Delta t}{\left(\Delta x^{n}\right)^{2}}, & \alpha_{m 5}=4-\frac{6 \Delta t}{\left(\Delta x^{n}\right)^{2}} \\
\alpha_{m 3}=1-x_{m}^{n} \frac{3 \Delta t(\dot{s})^{n}}{2 \Delta x^{n} s^{n}}-\frac{3 \Delta t}{\left(\Delta x^{n}\right)^{2}}, & \alpha_{m 6}=1+x_{m}^{n} \frac{3 \Delta t(\dot{s})^{n}}{2 \Delta x^{n} s^{n}}+\frac{3 \Delta t}{\left(\Delta x^{n}\right)^{2}}
\end{aligned}
$$

for finite element method based on variable space grid method. In this schemes, $s$ is the position of moving boundary, $\dot{s}$ is the velocity of moving boundary, $h^{n}\left(\equiv \Delta x^{n}\right)$ is mesh size and $k(\equiv \Delta t)$ is time step.

Furthermore, for finite element method based on variable space grid method (VSG-FEM), $s(t)$ is updated at each time step by using a suitable finite difference form of the Stefan condition $\frac{d s(t)}{d t}=-$ Ste $\frac{\partial U}{\partial x}$ on $x=s(t)$. Therefore we are going to use the following three point backward difference at the moving boundary

$$
\left.\frac{\partial U}{\partial x}\right|_{x=s(t)}=\frac{3 U_{N}-4 U_{N-1}+U_{N-2}}{2 \triangle x}+O(\triangle x)^{2}
$$

[14] and forward difference approximation for $\frac{d s(t)}{d t}$,

$$
\frac{d s(t)}{d t}=\frac{s^{n+1}-s^{n}}{\Delta t}+O(\Delta t)
$$

So, iterative relation of moving boundary is obtained as

$$
s^{n+1}=s^{n}-\text { Ste } \frac{\Delta t}{2 \Delta x^{n}}\left(3 U_{N}^{n}-4 U_{N-1}^{n}+U_{N-2}^{n}\right) \quad n=0,1,2, \ldots
$$


subject to

$$
s^{0}=0
$$

initial condition. So, the grid size $h^{n+1}=\frac{s^{n+1}}{N}$ is updated each time step where $N$ is the number of element. For the VSG-FEM, temperature distribution $U_{m}^{n} \sim U\left(x_{m}^{n}, t_{n}\right)$ is calculated at the points $x_{m}^{n}=m h^{n}, t_{n}=t_{0}+n k$ where $t_{0}$ is initial time.

\section{Boundary immobilisation method}

Boundary immobilisation method based on fixing moving boundary is a convenient method to solve Stefan problems. In this method, by using transformation of $x=\frac{\xi}{s(t)}$, moving interface $x=s(t)$ is turned into fixed boundary $\xi=1$, at new coordinate system $(\xi, t)$. This approach firstly was used by Landau [7] and applied to moving boundary problems by using finite difference and finite element methods in recent years [10,11].

In coordinate system $(\xi, t)$, reformulation equation of (1) can be written as

$$
\left.\frac{\partial U}{\partial t}\right|_{x}=\frac{\partial U}{\partial \xi} \frac{\partial \xi}{\partial t}+\left.\frac{\partial U}{\partial t}\right|_{\xi}=-\frac{x}{s^{2}(t)} \frac{d s}{d t} \frac{\partial U}{\partial \xi}+\left.\frac{\partial U}{\partial t}\right|_{\xi}
$$

So dimensionless Stefan problem becomes

$$
\frac{\partial U}{\partial t}=\frac{\xi}{s(t)} \frac{d s}{d t} \frac{\partial U}{\partial \xi}+\frac{1}{s^{2}(t)} \frac{\partial^{2} U}{\partial \xi^{2}}, \quad 0<\xi<1, \quad t>0
$$

subject to

$$
U(0, t)=1, \quad U(1, t)=0, \quad \frac{d s(t)}{d t}=-\frac{1}{s(t)} \frac{\partial U}{\partial \xi}, \quad \xi=1, \quad t>0
$$

boundary conditions and (4) initial condition. And exact solution (5) turns into equation

$$
U(\xi, t)=1-\frac{\operatorname{erf}(\xi \lambda)}{\operatorname{erf}(\lambda)}, 0 \leq \xi \leq 1, \quad t>0
$$

along with equations (6)-(7). Differently from variable space grid method Stefan condition takes the form at $\xi=1$

$$
s^{n+1}=s^{n}-\text { Ste } \frac{\Delta t}{2 \Delta \xi_{s}}\left(3 U_{N}^{n}-4 U_{N-1}^{n}+U_{N-2}^{n}\right), \quad n=0,1,2, \ldots
$$

with the aid of three point backward difference at the moving boundary in the transformed coordinate system $(\xi, t)$

$$
\left.\frac{\partial U}{\partial \xi}\right|_{\xi=1}=\frac{3 U_{N}-4 U_{N-1}+U_{N-2}}{2 \Delta \xi}+O(\Delta \xi)^{2}
$$

and (17) forward difference approximation for $\frac{d s(t)}{d t}$.

As the finite element method is applied to equation (19) which is obtained by using boundary immobilisation method subjected to (20) boundary conditions, we track similar way with variable space grid method. By using equation (10) defined in $(\xi, t)$ coordinate system for equation (19), we obtain

$$
\dot{\delta}_{m-1}+4 \dot{\delta}_{m}+\dot{\delta}_{m+1}=\xi_{m} \frac{(\dot{s})^{n}}{s^{n}} \frac{3}{\Delta \xi}\left(-\delta_{m-1}+\delta_{m+1}\right)+\frac{6 \alpha}{\left(s^{n} \Delta \xi\right)^{2}}\left(\delta_{m-1}-2 \delta_{m}+\delta_{m+1}\right)
$$


and by using Crank-Nicolson approach and time derivative given by equation (14) we obtain finite element system (15) which coefficients are

$$
\begin{array}{cc}
\alpha_{m 1}=1+\xi_{m} \frac{3 \Delta t(\dot{s})^{n}}{2 \Delta \xi s^{n}}-\frac{3 \Delta t}{\left(s^{n} \Delta \xi\right)^{2}}, & \alpha_{m 4}=1-\xi_{m} \frac{3 \Delta t(\dot{s})^{n}}{2 \Delta \xi s^{n}}+\frac{3 \Delta t}{\left(s^{n} \Delta \xi\right)^{2}} \\
\alpha_{m 2}=4+\frac{6 \Delta t}{\left(s^{n} \Delta \xi\right)^{2}}, & \alpha_{m 5}=4-\frac{6 \Delta t}{\left(s^{n} \Delta \xi\right)^{2}} \\
\alpha_{m 3}=1-\xi_{m} \frac{3 \Delta t(\dot{s})^{n}}{2 \Delta \xi s^{n}}-\frac{3 \Delta t}{\left(s^{n} \Delta \xi\right)^{2}}, & \alpha_{m 6}=1+\xi_{m} \frac{3 \Delta t(\dot{s})^{n}}{2 \Delta \xi s^{n}}+\frac{3 \Delta t}{\left(s^{n} \Delta \xi\right)^{2}}
\end{array}
$$

for BIM-FEM. In this schemes, $s$ is the position of moving boundary, $\dot{s}$ is the velocity of moving boundary, $h\left(\equiv \Delta \xi=\frac{1}{N}\right)$ is the grid size and $k(\equiv \Delta t)$ is time step. For BIM-FEM, temperature distribution $U_{m}^{n} \sim U\left(\xi_{m}, t_{n}\right)$ is calculated at the points $\xi_{m}=m h, t_{n}=t_{0}+n k$ where $t_{0}$ is initial time.

\section{Isotherm migration method (IMM)}

In the isotherm migration method, the temperature distribution $U$ can be interchanged with the space variable $x$, so that the solution is evaluated $x(U, t)$ instead of the more tridiational $U(x, t)$ [2]. This method was proposed by Chernousko [8] and Dix\&Cizek [9] who independently studied. This method provides constant boundaries to solve model problem. For this reason it is one of the most popular method for obtaining numerical solutions of moving boundary problems especially subjected to time dependent boundary conditions. Recently, Esen\&Kutluay [10] have obtained numerical solutions of the Stefan problem with Neumann boundary condition by using isotherm migration method constructed finite difference method.

By using the usual partial derivatives we have derivatives

$$
\frac{\partial U}{\partial x}=\left(\frac{\partial x}{\partial U}\right)^{-1}, \quad \frac{\partial^{2} U}{\partial x^{2}}=-\left(\frac{\partial x}{\partial U}\right)^{-3} \frac{\partial^{2} x}{\partial U^{2}}
$$

[2] and for the reason $d U=0$ we have

$$
\frac{\partial x}{\partial t}=-\frac{\partial U}{\partial t} \frac{\partial x}{\partial U} \text { or } \frac{\partial U}{\partial t}=-\frac{\partial x}{\partial t}\left(\frac{\partial x}{\partial U}\right)^{-1}
$$

After substituting equations (21)-(22) into heat equation (1), we obtain

$$
\frac{\partial x}{\partial t}=\left(\frac{\partial x}{\partial U}\right)^{-2} \frac{\partial^{2} x}{\partial U^{2}}, \quad 0<U<1, \quad t>0
$$

subject to

$$
x(0, t)=s(t), \quad x(1, t)=0, \quad \frac{d s(t)}{d t}=-S t e\left(\frac{\partial x}{\partial U}\right)^{-1}, \quad U=1, \quad t>0
$$

boundary and (4) initial condition. And the exact solution (5) becomes the following equation

$$
x(U, t)=2 \sqrt{t} \operatorname{erf}^{-1}((1-U) \operatorname{erf}(\lambda)), \quad 0 \leq U \leq 1, \quad t>0
$$

along with equations (6)-(7). Also by using three point forward difference

$$
\left.\frac{\partial x}{\partial U}\right|_{U=1}=\frac{-3 x_{0}+4 x_{1}-x_{2}}{2 \Delta U}+O(\Delta U)^{2}
$$


and (17) forward difference approximation for $\frac{d s(t)}{d t}$, Stefan condition takes the form at $U=1$

$$
s^{n+1}=s^{n}-\text { Ste } \frac{2(\Delta U)(\Delta t)}{-3 x_{0}^{n}+4 x_{1}^{n}-x_{2}^{n}}, \quad n=0,1, \ldots
$$

for isotherm migration method.

As the finite element method is applied to equation (23), the roles of dependent variable $U$ and independent space variable $x$ are changed in equation (10) given with nodal approximations. By substituting $x$ and its derivatives subjected to $U\left(x^{\prime}\right.$ and $\left.x^{\prime \prime}\right)$ into heat equation (1), we obtain

$$
\dot{\delta}_{m-1}+4 \dot{\delta}_{m}+\dot{\delta}_{m+1}=\frac{6\left(\delta_{m-1}-2 \delta_{m}+\delta_{m+1}\right)}{9\left(-\delta_{m-1}+\delta_{m+1}\right)^{2}} .
$$

And by using Crank-Nicolson approach and time derivative given by equation (14), we obtain finite element system (15) for the equation (23) in which coefficients are

$$
\begin{aligned}
& \alpha_{m 1}=1-3 Z(m), \alpha_{m 4}=1+3 Z(m) \\
& \alpha_{m 2}=4+6 Z(m), \alpha_{m 5}=4-6 Z(m) \\
& \alpha_{m 3}=1-3 Z(m), \alpha_{m 6}=1+3 Z(m)
\end{aligned}
$$

where $Z(m)=\frac{\Delta t}{9\left(\delta_{m+1}^{n}-\delta_{m-1}^{n}\right)^{2}}$ is non-linear term.

Numerical solutions of isotherm $x_{m}^{n} \sim x\left(U_{m}, t_{n}\right)$ are calculated at the points $U_{m}=m h, t_{n}=t_{0}+n k$, where $h\left(\equiv \Delta U=\frac{1}{N}\right)$ is the mesh size, $k(\equiv \Delta t)$ is time step and $t_{0}$ is initial time.

For the finite element methods based on variable space grid method, boundary immobilisation method and isotherm migration method, we obtain same finite element system (15) subjected to different coefficients. All of these methods have tridiagonal matrix system $N+1$ consisting of equations but $N+3$ unknowns. To solve the system uniquely two more equations are needed. We can obtain these equations from boundary conditions of the related method. And we use in the system to eliminate fictitious parameters $\delta_{-1}$ and $\delta_{N+1}$ for $m=0$ and $m=N$. So, the system can be demonstrated in matrix form

$$
A \delta^{n+1}=B \delta^{n}+r
$$

where $A, B$ are $(N+1) \times(N+1)$ tridiagonal matrixes and $r$ is a $(N+1)$ basing on boundary conditions. To start time evaluation of the approximate solution, $\delta^{0}$ must be determined, firstly.

Now, we consider determining of approximate solutions $\delta^{0}$ for variable space grid method. The others can be considered as the VSG-FEM. We take $U$ and $\xi$ the variables for BIM-FEM, $x$ and $U$ for IMM-FEM instead of $U$ and $x$ for VSG-FEM. To attain $\delta^{0}$ vector we require two conditions for $U_{N}\left(x, t_{0}\right)$

$$
U_{N}\left(x, t_{0}\right)=\sum_{m=-1}^{N+1} \delta_{m}^{0}(t) \phi_{m}(x) .
$$

(i) Initial condition $U\left(x, t_{0}\right)$ and $U_{N}\left(x, t_{0}\right)$ should be equal to each other for $N+1$ points.

(ii) To be able to solve the $A \delta^{0}=b$, we need two further equations which can be obtained from the first derivatives of end points of the domain.

After we find initial vector $\delta^{0}$, we get vectors $\delta^{1}, \delta^{2}, \ldots, \delta^{n}$ respectively. 


\section{Stability analysis}

We can use Von-Neumann method for VSG-FEM and BIM-FEM for the stability analysis. But, we can't use any stability analysis method for IMM-FEM because of the non-linear term $Z(m)$ presented in the finite element schemes of isotherm migration method.

In the Von-Neumann theory, the growth factor of typical Fourier mode is defined as

$$
\delta_{m}^{n}=\zeta^{n} e^{i m \beta h}
$$

where $\beta$ is the mode number and $h$ is element size. By substituting equation this equation into equation given with (15), and by performing some simplification operations we obtain

$$
\zeta=\frac{a_{1}+i b}{a_{2}-i b}
$$

where

$$
\begin{gathered}
a_{1}=\left(2+\frac{6 k}{h^{2}}\right) \cos \beta h+\left(4-\frac{6 k}{h^{2}}\right) \\
a_{2}=\left(2-\frac{6 k}{h^{2}}\right) \cos \beta h+\left(4+\frac{6 k}{h^{2}}\right) \\
b=\left(\frac{3 k}{h} \frac{(\dot{s})^{n}}{s^{n}} x_{m}^{n}\right) \sin \beta h
\end{gathered}
$$

for finite element method based on variable space grid and

$$
\begin{gathered}
a_{1}=\left(2+\frac{6 k}{h^{2}}\right) \cos \beta h+\left(4-\frac{6 k}{h^{2}}\right) \\
a_{2}=\left(2-\frac{6 k}{h^{2}}\right) \cos \beta h+\left(4+\frac{6 k}{h^{2}}\right) \\
b=\left(\frac{3 k}{h} \frac{(\dot{s})^{n}}{s^{n}} \xi_{m}\right) \sin \beta h
\end{gathered}
$$

for finite element method based on boundary immobilisation method. For the stability of these methods, the growth factor must satisfy $|\zeta| \leq 1$, so $\left|\frac{a_{1}+i b}{a_{2}-i b}\right| \leq 1$ if only if $a_{1}^{2} \leq a_{2}^{2}$. If we do essential processing we will see that the system of (15) is unconditionally stable for VSG-FEM and BIM-FEM. It should be pointed that $h$ shows $\Delta x^{n}$ for VSG-FEM and $\zeta$ for BIM-FEM and $k$ shows $\Delta t$ for both of the methods.

\section{Numerical results and conclusion}

In this section, we have demonstrated the numerical results obtained by using VSG-FEM, BIM-FEM and IMM-FEM for temperature distribution, the position of moving boundary and the velocity of the moving boundary. We have compared the present solutions and other numerical solutions obtained by using FDM and NIM $\left(N_{x}=10\right)$ [12]. Due to the singularity at the $t_{0}=0$, we use $t_{0}=0.01$ to initialize the numerical procedure. To compare the other numerical solutions obtained by using finite difference, we use a constant grid number $N=10$ and the time step $\Delta t=0.000002$ which are used in reference [12]. 
It should be noted that we use two different Ste numbers (Ste $=0.2$ and Ste $=1.0$ ) for the computational results. And the values of $\lambda$ corresponded to related Ste $=0.2$ and Ste $=1.0$ numbers are obtained by using the transcendental equation (8), which are $\lambda=0.30642$ and $\lambda=0.62006$, respectively.

Table 2: Comparison of temperature distribution with results from [12] for Ste $=0.2$ and $t_{f}=1.6$.

\begin{tabular}{llllllll}
\hline$x / s$ & VSG-FEM & Rel. Err. \% & NIM [12] & Rel. Err. \% & FDM [12] & Rel. Err. \% & Exact Solution \\
\hline 0.0 & 1.0 & 0.0 & 1.0 & 0.0 & 1.0 & 0.0 & 1.0 \\
0.1 & 0.896893 & 0.000000 & 0.896893 & 0.000000 & 0.896891 & 0.000223 & 0.896893 \\
0.2 & 0.793979 & 0.000126 & 0.793979 & 0.000126 & 0.793976 & 0.000504 & 0.793980 \\
0.3 & 0.691451 & 0.000145 & 0.691451 & 0.000145 & 0.691446 & 0.000868 & 0.691452 \\
0.4 & 0.589500 & 0.000000 & 0.589498 & 0.000339 & 0.589493 & 0.001187 & 0.589500 \\
0.5 & 0.488310 & 0.000204 & 0.488309 & 0.000410 & 0.488303 & 0.001638 & 0.488311 \\
0.6 & 0.388067 & 0.000257 & 0.388066 & 0.000515 & 0.388060 & 0.002061 & 0.388068 \\
0.7 & 0.288946 & 0.000346 & 0.288945 & 0.000692 & 0.288940 & 0.002423 & 0.288947 \\
0.8 & 0.191120 & 0.000523 & 0.191120 & 0.000523 & 0.191115 & 0.003139 & 0.191121 \\
0.9 & 0.094753 & 0.000000 & 0.094752 & 0.001055 & 0.094750 & 0.003166 & 0.094753 \\
1.0 & 0.0 & 0.0 & 0.0 & 0.0 & 0.0 & 0.0 & 0.0 \\
\hline
\end{tabular}

In Table 2, the present computational results for VSG-FEM and the other numerical results obtained by the other researchers are compared for temperature distribution. Our numerical solutions are more accurate than the others and in good agreement with the exact solution for $S t e=0.2$.

Table 3: Comparison of numerical solutions for the position of the moving boundary $s(t)$ with results from [12] for Ste $=0.2$ at different time values.

\begin{tabular}{ccccccc}
\hline$t_{f}$ & VSG-FEM & BIM-FEM & IMM-FEM & NIM [12] & FDM [12] & Exact Solution \\
\hline 0.2 & 0.27414 & 0.27414 & 0.27414 & 0.27409 & 0.27414 & 0.27407 \\
0.4 & 0.38770 & 0.38770 & 0.38784 & 0.38763 & 0.38769 & 0.38759 \\
0.6 & 0.47483 & 0.47483 & 0.47489 & 0.47474 & 0.47482 & 0.47470 \\
0.8 & 0.54829 & 0.54829 & 0.54836 & 0.54819 & 0.54828 & 0.54814 \\
1.0 & 0.61301 & 0.61301 & 0.61308 & 0.61289 & 0.61300 & 0.61284 \\
1.2 & 0.67152 & 0.67152 & 0.67160 & 0.67139 & 0.67150 & 0.67133 \\
1.4 & 0.72532 & 0.72532 & 0.72532 & 0.72541 & 0.72531 & 0.72512 \\
1.6 & 0.77540 & 0.77540 & 0.77550 & 0.77525 & 0.77539 & 0.77519 \\
\hline \hline$t_{f}$ & Rel. Err. \% & Rel. Err. \% & Rel. Err. \% & Rel. Err. \% & Rel. Err. \% & \\
\hline 0.2 & 0.02554 & 0.02554 & 0.02554 & 0.00730 & 0.02554 & \\
0.4 & 0.02838 & 0.02838 & 0.06450 & 0.01032 & 0.02580 & \\
0.6 & 0.02738 & 0.02738 & 0.04002 & 0.00843 & 0.02528 & \\
0.8 & 0.02737 & 0.02737 & 0.04013 & 0.00912 & 0.02554 & \\
1.0 & 0.02773 & 0.02773 & 0.03916 & 0.00816 & 0.02611 & \\
1.2 & 0.02830 & 0.02830 & 0.04022 & 0.00894 & 0.02532 & \\
1.4 & 0.02758 & 0.02758 & 0.02758 & 0.00827 & 0.02620 & \\
1.6 & 0.02709 & 0.02709 & 0.03999 & 0.00774 & 0.02580 & \\
\hline
\end{tabular}

In Table 3 and Table 4, the position of moving boundary and its velocity are given together with percentage relative errors for Ste $=0.2$. As the nodal integral method applies, each of node number the heat equation (1) are represented differential equations system. As a result of this, for increasing the node number it is difficult to get the numerical 
solutions of NIM. But we easily obtain closer numerical solutions of the position of the moving boundary and its velocity for the larger element numbers as given in the Table 5.

In Table 5, we present the position of moving boundary and the velocity of moving boundary for the larger element numbers, Ste $=0.2$ and $t_{f}=1.6$. The obtained numerical results show that by the increasing mesh sizes three different finite element method based on cubic B-splines, VSG-FEM, BIM-FEM and IMM-FEM attain to expected convergence.

Table 4: Comparison of numerical solutions for the velocity of the moving boundary $\dot{s}(t)$ with results from [12] for Ste $=0.2$ at different time values.

\begin{tabular}{ccccccc}
\hline$t_{f}$ & VSG-FEM & BIM-FEM & IMM-FEM & NIM [12] & FDM [12] & Exact Solution \\
\hline 0.2 & 0.68538 & 0.68537 & 0.68546 & 0.68524 & 0.68536 & 0.68518 \\
0.4 & 0.48463 & 0.48463 & 0.48469 & 0.48454 & 0.48462 & 0.48449 \\
0.6 & 0.39570 & 0.39570 & 0.39575 & 0.39562 & 0.39569 & 0.39559 \\
0.8 & 0.34268 & 0.34268 & 0.34273 & 0.34262 & 0.34268 & 0.34259 \\
1.0 & 0.30651 & 0.30651 & 0.30654 & 0.30645 & 0.30650 & 0.30642 \\
1.2 & 0.27980 & 0.27980 & 0.27983 & 0.27975 & 0.27979 & 0.27972 \\
1.4 & 0.25904 & 0.25904 & 0.25904 & 0.25908 & 0.25904 & 0.25897 \\
1.6 & 0.24231 & 0.24231 & 0.24234 & 0.24227 & 0.24231 & 0.24225 \\
\hline \hline$t_{f}$ & Rel.Err.\% & Rel.Err.\% & Rel.Err.\% & Rel.Err.\% & Rel.Err.\% & \\
\hline 0.2 & 0.02919 & 0.02773 & 0.04086 & 0.00876 & 0.02627 & \\
0.4 & 0.02890 & 0.02890 & 0.04128 & 0.01032 & 0.02683 & \\
0.6 & 0.02781 & 0.02781 & 0.04044 & 0.00758 & 0.02528 & \\
0.8 & 0.02627 & 0.02627 & 0.04087 & 0.00876 & 0.02627 & \\
1.0 & 0.02937 & 0.02937 & 0.03916 & 0.00979 & 0.02611 & \\
1.2 & 0.02860 & 0.02860 & 0.03932 & 0.01073 & 0.02503 & \\
1.4 & 0.02703 & 0.02703 & 0.02703 & 0.00772 & 0.02703 & \\
1.6 & 0.02477 & 0.02477 & 0.03715 & 0.00826 & 0.02477 & \\
\cline { 1 - 5 }
\end{tabular}

In Table 6, the numerical results of temperature distribution obtained by using VSG-FEM are compared NIM and FDM for Ste $=1.0$ and $t_{f}=0.8$. Computational results have smaller percentage errors than FDM. So, it can be said that with increasing element number, temperature distribution will be expected, compared to FDM and NIM [12].

Table 5: The position and velocity of moving boundary for different element numbers and Ste $=0.2, t_{f}=1.6$.

\begin{tabular}{lllllll}
\hline Method & $N=10$ & $N=20$ & $N=40$ & $N=80$ & Exact Solution & \\
\hline VSG-FEM & 0.77540 & 0.77525 & 0.77521 & 0.77520 & & \\
BIM-FEM & 0.77540 & 0.77525 & 0.77521 & 0.77520 & 0.77519 & $s(t)$ \\
IMM-FEM & 0.77550 & 0.77528 & 0.77522 & 0.77520 & & \\
\hline \hline VSG-FEM & 0.24231 & 0.24226 & 0.24225 & 0.24225 & & \\
BIM-FEM & 0.24231 & 0.24227 & 0.24225 & 0.24225 & 0.24225 & $\dot{s}(t)$ \\
IMM-FEM & 0.24234 & 0.24227 & 0.24226 & 0.24225 & & \\
\hline
\end{tabular}


Table 6: Comparison of temperature distribution with results from [12] for Ste $=1.0$ and $t_{f}=0.8$.

\begin{tabular}{llllllll}
\hline$x / s$ & VSG-FEM & Rel. Err. \% & NIM [12] & Rel. Err. \% & FDM [12] & Rel. Err. \% & Exact Solution \\
\hline 0.0 & 1.0 & 0.0 & 1.0 & 0.0 & 1.0 & 0.0 & 1.0 \\
0.1 & 0.887205 & 0.000902 & 0.887190 & 0.000789 & 0.887180 & 0.001916 & 0.887197 \\
0.2 & 0.775274 & 0.002193 & 0.775245 & 0.001548 & 0.775224 & 0.004257 & 0.775257 \\
0.3 & 0.665049 & 0.003609 & 0.665007 & 0.002707 & 0.664978 & 0.007067 & 0.665025 \\
0.4 & 0.557331 & 0.004665 & 0.557283 & 0.003948 & 0.557248 & 0.010228 & 0.557305 \\
0.5 & 0.452876 & 0.006846 & 0.452822 & 0.005079 & 0.452783 & 0.013691 & 0.452845 \\
0.6 & 0.352353 & 0.008514 & 0.352300 & 0.006528 & 0.352260 & 0.017881 & 0.352323 \\
0.7 & 0.256357 & 0.010143 & 0.256310 & 0.008193 & 0.256173 & 0.022627 & 0.256331 \\
0.8 & 0.165386 & 0.030241 & 0.165350 & 0.009676 & 0.165320 & 0.027817 & 0.165336 \\
0.9 & 0.079836 & 0.013780 & 0.079816 & 0.011275 & 0.079798 & 0.033824 & 0.079825 \\
1.0 & 0.0 & 0.0 & 0.0 & 0.0 & 0.0 & 0.0 & 0.0 \\
\hline
\end{tabular}

In Table 7 and Table 8, the position of the moving boundary and its velocity are given together with percentage relative errors for Ste $=1.0$. NIM has the more accurate solution in tables, but we can obtain closer numerical solutions of the temperature distribution, the position of the moving boundary and its velocity for the larger element numbers than given in the Table 7 and Table 8. Since the moving boundary moves faster for larger Ste numbers, the Stefan problems get difficult for larger Stefan numbers. This can be shown both for temperature distribution, the position of moving boundary and its velocity for Ste $=1.0$ compared to those obtained for Ste $=0.2$.

In Table 9, we show the position of moving boundary and the velocity of moving boundary for the larger element numbers, Ste $=1.0$ and $t_{f}=0.8$. It is clearly seen that newly obtained numerical solutions of the position of moving boundary and the velocity of moving boundary are so close to exact solutions for the larger element number.

Table 7: Comparison of numerical solutions for the velocity of the moving boundary $s(t)$ with results from [12] for Ste $=1.0$ at different time values.

\begin{tabular}{ccccccc}
\hline$t_{f}$ & VSG-FEM & BIM-FEM & IMM-FEM & NIM [12] & FDM [12] & Exact Solution \\
\hline 0.1 & 0.39235 & 0.39235 & 0.39302 & 0.39224 & 0.36226 & 0.39216 \\
0.2 & 0.55488 & 0.55489 & 0.55589 & 0.55473 & 0.55474 & 0.55460 \\
0.3 & 0.67960 & 0.67960 & 0.68085 & 0.67941 & 0.67942 & 0.67924 \\
0.4 & 0.78474 & 0.78474 & 0.78619 & 0.78451 & 0.78453 & 0.78432 \\
0.5 & 0.87736 & 0.87737 & 0.87900 & 0.87711 & 0.87713 & 0.87690 \\
0.6 & 0.96111 & 0.96111 & 0.96260 & 0.96083 & 0.96085 & 0.96060 \\
0.7 & 1.03811 & 1.03812 & 1.04006 & 1.03782 & 1.03784 & 1.03756 \\
0.8 & 1.10979 & 1.10979 & 1.11187 & 1.10948 & 1.10950 & 1.10920 \\
\hline \hline$t_{f}$ & Rel. Err. \% & Rel. Err. \% & Rel. Err. \% & Rel. Err. \% & Rel. Err. \% & \\
\hline 0.1 & 0.04844 & 0.04844 & 0.21930 & 0.02040 & 0.02550 & \\
0.2 & 0.05049 & 0.05229 & 0.23260 & 0.02344 & 0.02524 & \\
0.3 & 0.05300 & 0.05300 & 0.23703 & 0.02503 & 0.02650 & \\
0.4 & 0.05355 & 0.05355 & 0.23842 & 0.02422 & 0.02677 & \\
0.5 & 0.05246 & 0.05360 & 0.23948 & 0.02395 & 0.02623 & \\
0.6 & 0.05310 & 0.05310 & 0.20820 & 0.02394 & 0.02603 & \\
0.7 & 0.05301 & 0.05397 & 0.24095 & 0.02506 & 0.02699 & \\
0.8 & 0.05319 & 0.05319 & 0.24071 & 0.02524 & 0.02705 & \\
\hline
\end{tabular}


Table 8: Comparison of numerical solutions for the velocity of the moving boundary $\dot{s}(t)$ with results from [12] for Ste $=1.0$ at different time values .

\begin{tabular}{ccccccc}
\hline$t_{f}$ & VSG-FEM & BIM-FEM & IMM-FEM & NIM [12] & FDM [12] & Exact Solution \\
\hline 0.1 & 1.96198 & 1.96196 & 1.96604 & 1.96140 & 1.96137 & 1.96080 \\
0.2 & 1.38729 & 1.38728 & 1.39004 & 1.38689 & 1.38688 & 1.38650 \\
0.3 & 1.13271 & 1.13270 & 1.13492 & 1.13238 & 1.13238 & 1.13207 \\
0.4 & 0.98095 & 0.98094 & 0.98285 & 0.98066 & 0.98067 & 0.98040 \\
0.5 & 0.87738 & 0.87738 & 0.87908 & 0.87713 & 0.87714 & 0.87690 \\
0.6 & 0.80094 & 0.80093 & 0.80248 & 0.80071 & 0.80071 & 0.80049 \\
0.7 & 0.74152 & 0.74152 & 0.74295 & 0.74131 & 0.74132 & 0.74111 \\
0.8 & 0.69363 & 0.69363 & 0.69496 & 0.69343 & 0.69344 & 0.69325 \\
\hline \hline$t_{f}$ & Rel. Err. \% & Rel. Err. \% & Rel. Err. \% & Rel. Err. \% & Rel. Err. \% & \\
\hline 0.1 & 0.06018 & 0.05916 & 0.26724 & 0.03060 & 0.02907 & \\
0.2 & 0.05698 & 0.05626 & 0.25532 & 0.02813 & 0.02741 & \\
0.3 & 0.05653 & 0.05565 & 0.25736 & 0.02738 & 0.02738 & \\
0.4 & 0.05610 & 0.05610 & 0.24990 & 0.02652 & 0.02754 & \\
0.5 & 0.05474 & 0.05474 & 0.24860 & 0.02623 & 0.02737 & \\
0.6 & 0.05621 & 0.05622 & 0.24860 & 0.02748 & 0.02548 & \\
0.7 & 0.05532 & 0.05532 & 0.24828 & 0.02699 & 0.02834 & \\
0.8 & 0.05481 & 0.05481 & 0.24666 & 0.02596 & 0.02741 & \\
\hline
\end{tabular}

Table 9: The position and velocity of moving boundary for different element numbers and Ste $=1.0, t_{f}=0.8$.

\begin{tabular}{lllllll}
\hline Method & $N=10$ & $N=20$ & $N=40$ & $N=80$ & Exact Solution & \\
\hline VSG-FEM & 1.10979 & 1.10933 & 1.10923 & 1.10921 & & $s(t)$ \\
BIM-FEM & 1.10979 & 1.10933 & 1.10926 & 1.10921 & 1.10920 & \\
IMM-FEM & 1.11187 & 1.10999 & 1.10942 & 1.10926 & & $\dot{s}(t)$ \\
\hline \hline VSG-FEM & 0.69363 & 0.69333 & 0.69327 & 0.69326 & & \\
BIM-FEM & 0.69363 & 0.69333 & 0.69329 & 0.69325 & 0.69325 & \\
IMM-FEM & 0.69396 & 0.69376 & 0.69376 & 0.69329 & & \\
\hline
\end{tabular}

In this manuscript, finite element method constructed with VSG, BIM and IMM was applied to melting problem described on semi-infinite domain. The computational results was compared with the numerical solutions obtained by using finite difference method and nodal integral method. The present numerical results of temperature distribution have more accurate solution than FDM and NIM for Ste $=0.2$. The newly numerical solutions for temperature distribution, the position of moving boundary and the velocity of the moving boundary are in good agreement with the exact solutions. It was shown that with increasing element numbers the numerical solutions converge to exact solutions for the position of moving boundary and the velocity of moving boundary. It can be seen the tables finite element methods based on VSG, BIM and IMM reasonably more accurate solutions for small values of Stefan numbers and larger numbers of elements. For the large numbers of elements, finite element methods can be applied the Stefan problem easily to attain a high degree of accuracy.

\section{Competing interests}

The authors declare that they have no competing interests. 


\section{Authors' contributions}

All authors have contributed to all parts of the article. All authors read and approved the final manuscript.

\section{References}

[1] J. Stefan, Uber die theorie der eisbildung inbesondee uber die eisbindung im polarmeere, Ann. Phys. U. Chem. 42 (1891) $269-286$.

[2] J. Crank, Free and Moving Boundary Problems, Clarendon Press, Oxford, 1984.

[3] W.D. Murray, and F. Landis, Numerical and Machine Solutions of Transient Heat Conduction Involving Melting or Freezing, J. Heat Transfer 81 106-112 (1959).

[4] S. Kutluay, A.R. Bahadir, A. Ozdes, The numerical solution of one-phase classical Stefan problem, J. Comp. Appl. Math. 81 (1997) 35-44.

[5] N. S. Asaithambi, A Variable Time-Step Galerkin Method for the One- Dimensional Stefan Problem, Applied Mathematics and Computation 81 (1997) 189-200.

[6] T.R.Goodman, The Heat-Balance Integral and its Application to Problems Involving a Change of Phase, Trans. ASME 80 (1959) 335-342.

[7] H.G. Landau, Heat conduction in a melting solid, Quart. J. Appl. Math. 8 (1950) 81-94.

[8] F.L. Chernousko,, Solution of non-linear Problems in Medium with Changes, Int. Chem. Engng. 10 (1970) 42-48.

[9] R.C. Dix and J. Cizek, The isotherm migration method for transient heat conduction analysis, in: U. Grigull and E. Hahne, Eds., Heat Transfer 1, Proc. 4th Znternat. Heat Transfer Conf, Paris (Elsevier, Amsterdam, 1970) Cul.1.

[10] A. Esen and S.Kutluay, An isotherm migration formulation for one-phase Stefan problem with a time dependent Neumann condition, Applied Mathematics and Computation 150 (2004) 59-67.

[11] A. Esen, S. Kutluay, A numerical solution of the Stefan problem with a Neumann-type boundary condition by enthalpy method, Applied Mathematics and Computation 148 (2004) 321-329.

[12] J. Caldwell, S. Savovic', Y.Y. Kwan, Nodal Integral and Finite Difference Solution of One-Dimensional Stefan Problem, J. Heat Mass Trans. 125 (2003) 523-527.

[13] A.H.A. Ali, G.A. Gardner, L.R.T. Gardner, A collocation solution for Burgers equation using cubic B-spline finite elements, Comput. Meth. Appl. Mech. Eng. 100 (1992) 325-337.

[14] R.M. Furzeland, A comparative study of numerical methods for moving boundary problems, J. Inst. Maths. Appl. 26 (1980) 411-429. 